\title{
Facile, high-yield, regioselective synthesis of ortho-nitrophenols using cerium (IV) ammonium nitrate
}

\author{
Ramadas Sathunuru, U. Narasimha Rao, and Ed Biehl* \\ Chemistry Department, Southern Methodist University, Dallas, TX 75275 \\ E-mail: ebiehl@mail.smu.edu
}

(received 09 Oct 03; accepted 30 Jan 04; published on the web 05 Feb 04)

\begin{abstract}
Certain phenols possessing at least one unsubstituted ortho position have been found to undergo rapid, regioselective ortho nitration with CAN (cerium (IV) ammonium nitrate) in the presence

of $\mathrm{NaHCO}_{3}$ at room temperature to yield $o$-nitrophenols in high yields. Substituents tolerating these nitration conditions ranged from the activating methoxy and methyl groups to the moderately deactivating $\mathrm{Cl}, \mathrm{Br}, \mathrm{CHO}$ and $\mathrm{CO}_{2} \mathrm{Me}$ groups. In contrast, phenols that contained a strongly deactivating group such as nitro or cyano or 2,6-disubstituted phenols were not nitrated by the $\mathrm{CAN} / \mathrm{NaHCO}_{3}$ reagent. More complex nitrophenols such as 6-hydroxy-5-nitro-1,3benzoxanthiol-2-one, 7-hydroxy-6-nitro-3,4,8-trimethylcoumarin, 6-hydroxy-5-flavanone, 1-(4hydroxy-3-nitrophenyl)- $1 H$-tetrazole-5-thiol, 2-(2-hydroxy-3-nitrophenyl)benzoxazole were also prepared in good yields by the $\mathrm{CAN} / \mathrm{NaHCO}_{3}$ reagent.
\end{abstract}

Keywords: Phenol, nitration, cerium(IV)ammoniumnitrate

\section{Introduction}

Nitrations of phenols using the classical method of nitric acid in sulphuric acid generally gives complex mixtures containing $o$ - and $p$-nitrophenols, dinitrated phenols, plus inextractible tars of phenolic oxidation products. ${ }^{1}$ Consequently, alternative nitration methods have been sought to accomplish clean and regioselective mononitration of phenols. Some of these include the use of: (1) a two-phase system (ether-phenol/water- $\left.\mathrm{NaNO}_{3}-\mathrm{NaNO}_{2}-\mathrm{H}^{+}\right),{ }^{2}$ (2) an ionic complex of $\mathrm{N}_{2} \mathrm{O}_{4}$ with 18-crown-6, ${ }^{3}$ (3) nitrosation-oxidation with metallic $\mathrm{HSO}_{4}{ }^{-}$and $\mathrm{NaNO}_{3}{ }^{4}$ and (4) nitrocyclohexadienone as the nitrating agent, ${ }^{5}$ etc. While these methods give clean mononitration of phenols, both $o$ and $p$-nitrophenols are obtained. $o$-Nitrophenols are valuable precursors to a variety of biologically important heterocycles such as 2,3-dihydro-2H-1,4-benzoxoin-3(4H)ones, ${ }^{6}$ and benzoxazoles. ${ }^{7}$ Thus, a convenient method for the regioselective introduction of an ortho nitro group onto phenols is desirable. A survey of the literature shows the lack of $100 \%$ ortho nitration, however, there are a few reports of almost regioselective ortho nitration of phenols. Two of these involved passing $\mathrm{NO}_{2}-\mathrm{N}_{2} \mathrm{O}_{4}$ gas into a solution of phenol and pyridinium 
carboxylic $\operatorname{acid}^{8}$ or vigorously stirring a suspension of phenol with "claycop" (an acidic montmorillonite clay impregnated with anhydrous cupric nitrate). ${ }^{9}$ These methods gave mixtures of $o$ - and $p$-nitrophenols with high $o / p$ ratios of $95: 5$ and 86:6, respectively.

\section{Results and Discussion}

Recently, 7-hydroxycoumarins have been nitrated with CAN and $\mathrm{H}_{2} \mathrm{O}_{2}$ as co-oxidants in water to give a 9:1 mixture of 7-hydroxy- 6-nitro- and 7-hydroxy-6,8-dinitrocoumarins. ${ }^{10}$ However, nitration of simple phenols with the $\mathrm{CAN} / \mathrm{H}_{2} \mathrm{O}_{2}$ reagent was found to produce mixtures of nitrophenols as well as hydroxylation and coupling products. ${ }^{11,12}$ When CAN is coated on silica $\left(\mathrm{CAN} / \mathrm{SiO}_{2}\right)$, a milder nitrating reagent is obtained which has been used in the nitration of, polynuclear, ${ }^{13}$ heterocyclic ${ }^{14}$ and electron-rich aromatic compounds.${ }^{15}$ In the latter case, mixtures of $o$ - and $p$-isomers were obtained.

In an unrelated study, $\mathrm{CAN}$ in the presence of $\mathrm{NaHCO}_{3}$ and acetonitrile was found to mediate the oxidative coupling between thiophenols and aromatic nitriles affording benzothiazoles. ${ }^{16}$ It occurred to us, that the replacement of $\mathrm{H}_{2} \mathrm{O}_{2}$ with $\mathrm{NaHCO}_{3}$ might result in a more selective CAN nitrating reagent with diminished oxidative capability. Thus, we have treated a variety of phenols, having at least one unsubstituted ortho position with $\mathrm{CAN} / \mathrm{NaHCO}_{3}$ and , as shown in Table 1, obtained o-nitrophenols in all cases. Best yields of the $o$-nitrophenols were attained by carrying out the reactions in $\mathrm{MeCN}$ at room temperature for $30 \mathrm{~min}$. The absence of $p$-nitrophenols as well as hydroxylation and coupling products was ascertained by $\mathrm{GC} / \mathrm{MS}$ analysis of the crude reaction mixtures.

The wide scope of this nitration can be appreciated by discussing the different classes of phenols separately. As shown in Table 1, a wide variety of monocyclic phenols (1a-o) possessing $\mathrm{Me}, \mathrm{OMe}, \mathrm{Cl}, \mathrm{Br}, \mathrm{CO}_{2} \mathrm{Et}$, and $\mathrm{CHO}$ substituents reacted with $\mathrm{CAN} / \mathrm{NaHCO}_{3}$ to give the corresponding $o$-nitrophenols

Table 1. Yields ${ }^{\mathrm{a}}$ of nitrophenols (2a-v from the $\mathrm{CAN} / \mathrm{NaHCO}_{3}$ nitration of phenols (1a-v)

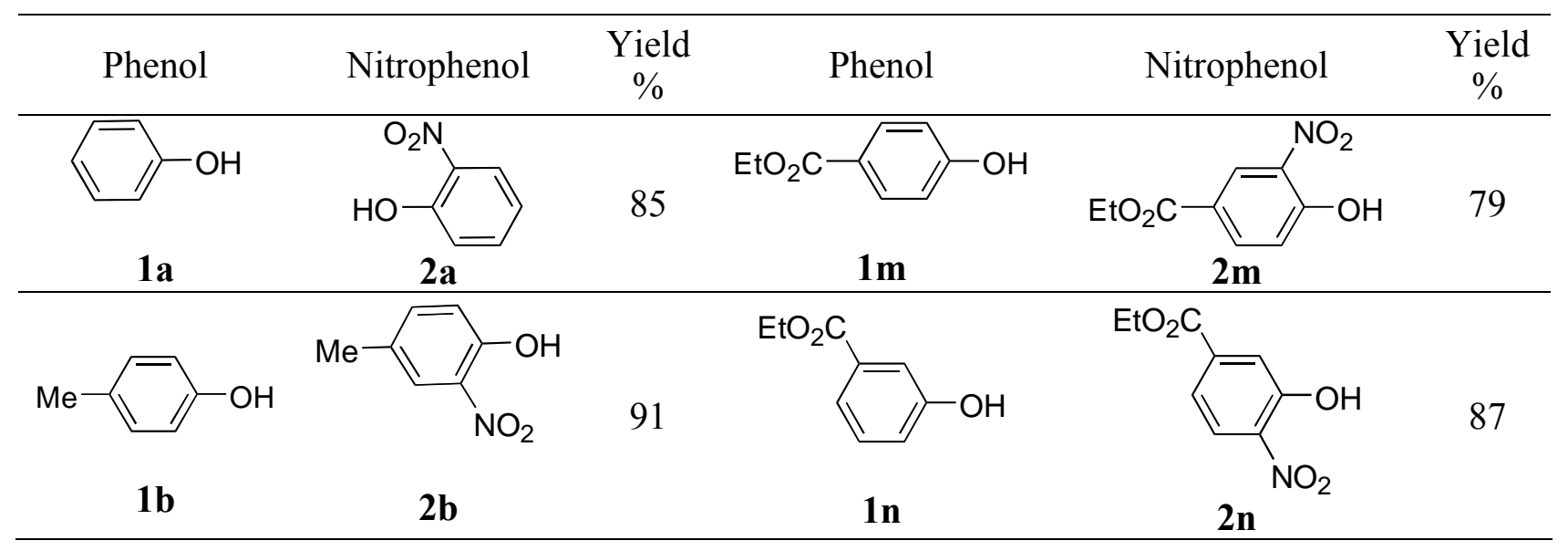


Table 1. Continued

(n)


Table 1. Continued

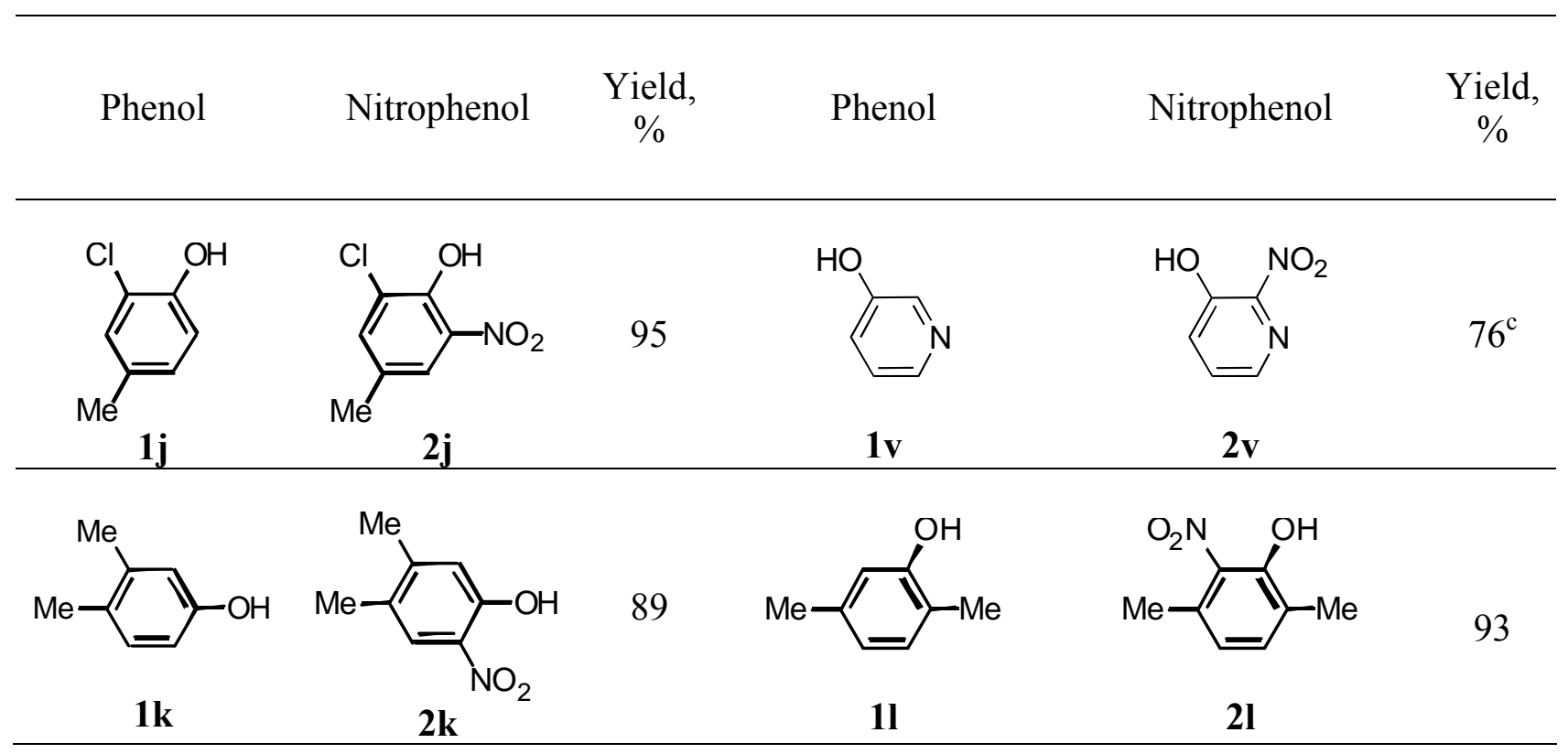

${ }^{a}$ isolated yields. ${ }^{b} 20 \%$ yield of benzoquinone. ${ }^{c}$ stirring for $6 \mathrm{~h}$.

(2a-o), exclusively. In all cases, ${ }^{1} \mathrm{H}$ NMR and ${ }^{13} \mathrm{C}$ NMR and GC/MS analysis confirmed the structure and purity of the isolated products.

The yields of the monocyclic phenols (2a-o) ranged from $72 \%$ to $95 \%$, which are, for the most part, significantly higher than any previously reported. For example, phenol (1a) was converted to 2-nitrophenol (2a) in $85 \%$ yield with $\mathrm{CAN} / \mathrm{NaHCO}_{3}$, while the $\mathrm{CAN} / \mathrm{H}_{2} \mathrm{O}_{2}$ reagent gave 2a in only $15 \%$ yield. ${ }^{17}$ Additionally, $\mathrm{CAN} / \mathrm{NaHCO}_{3}$ treatment of $p$-cresol (1b) gave 4methyl-2-nitrophenol (2b) as single product in $95 \%$ yield, compared to the $30 \%$ yield from similar treatment with $\mathrm{CAN} / \mathrm{H}_{2} \mathrm{O}_{2}$ using dodecyl sodium sulfate as a surfactant ${ }^{7}$ and the $60 \%$ yield obtained when $\mathbf{1 b}$ was first impregnated on $\mathrm{SiO}_{2 .}{ }^{18}$ In addition, other 4-substituted phenols, i.e. p-bromo- (1c), p-methoxy- (1d), and ethyl p-hydroxybenzoate acid (1m) underwent nitration with the $\mathrm{CAN} / \mathrm{NaHCO}_{3}$ reagent to give 4-bromo-2-nitrophenol (2c), 4-methoxy-2nitrophenol (2d) and ethyl 4-hydroxy-3-nitrobenzoate (2m) in $86 \%, 79 \%$ and $72 \%$ yields, respectively. The yield of $\mathbf{2 d}$ (72\%) was unfavorably affected by partial oxidation of $\mathbf{1 d}$ to $p$ benzoquinone (20\%). Interestingly $\mathbf{1 d}$ was quantitatively oxidized to $p$-quinone by $\mathrm{CAN} / \mathrm{SiO}_{2} .{ }^{15}$ 4-hydroxy- and 4-aminophenols (not shown in Table 1) did not undergo nitration under similar conditions, but rather gave mixtures of oxidized products.

Table 1 also lists the results for the nitration of 3-substituted phenols, i.e. 3-methoxy-(1e), 3bromo- (1) $)$ 3-methyl- (1g) and 3-chlorophenol (1h) and (1e-h), as well as ethyl 3hydroxybenzoate (1n) and 3-hydroxybenzaldehyde (10). With the exception of 1e, the 3substituted derivatives were nitrated regioselectively at the unhindered site (C-6, in the case of the 3-substituted phenols and C-4, in the case of the 3-hydroxy derivatives) to give the 5substituted 2-nitrophenols (2f-h), 3-hydroxy-4-nitro benzoate (2n) and 3-hydroxy-4- 
nitrobenzaldehyde (2o). The yields ranged from $87 \%$ to $93 \%$. With the exception of methyl, the aforementioned substituents are -I groups (electron-withdrawing by induction) and thus would be expected to discourage electrophilic addition at the hindered site. Steric factor for these substituents as well as weakly activating methyl should also impede addition at the hindered site. In the exceptional case, 3-methoxyphenol (1e) underwent CAN nitration exclusively at the hindered C-2 site giving 3-methoxy-2-nitrophenol (2e) in 90\% yield. This anomalous result indicates that the strong mesomeric effect of methoxy activates the hindered carbon site. Not only are the previous yields of substituted nitrophenols lower that those reported here, but most preparations involve at least two steps. For example, the highest yield of $\mathbf{2 e}$ previously reported was $70 \%$ using a two-step process of nitrosation of $1 \mathbf{e}$ followed by nitric acid oxidation. ${ }^{19}$ Importantly, 3-hydroxybenzaldehyde (10) and 3-methoxyphenol (1e) were not oxidized using the $\mathrm{CAN} / \mathrm{NaHCO}_{3}$ reagent.

The mild nitrating power of CAN was further shown by the selective ortho mononitration of the two dimethylphenol derivatives 3,4-dimethylphenol (1k) and 2,5-dimethylphenol (11), which gave 2-nitro-4,5-dimethylphenol (2j) and 2-nitro-3.6-dimethylphenol (2l) in 89\% and 93\% yields, respectively. Previously, dialkyl phenols, when treated with nitrating agents such as $\mathrm{NO}_{2}{ }^{20}$ were found to give only polynitrophenols and 2,5-cyclohexadienones.

The results in Table 1 show that the $\mathrm{CAN} / \mathrm{NaHCO}_{3}$ reagent can also nitrate certain polycyclic phenols regioselectively. For example, 1-naphthol (1p) was converted to 2-nitro-1-naphthol (2p) in $85 \%$ yield, whereas treatment with nitrocyclohexadienone gave mixtures of 2- and 4-nitro-1napthols. ${ }^{21}$ Likewise, CAN nitration of 2-naphthol (1q) afforded 1-nitro-2-naphthol (2q) in 72\% yield which is substantially greater that the highest yield (49\%) previously reported. ${ }^{22}$ More complex nitrophenols such as 7-hydroxy-6-nitro-3,4,8-trimethylcoumarin (2r), 6-hydroxy-5nitro-1,3-benzothio-2-one (2s) and 2-(2-hydroxy-3-nitrophenyl)benzoxazole (2t) were prepared in good yields from the corresponding phenols. Interestingly, treatment of 2-(4-hydroxyphenyl)5-pyrimidinol (1u) with CAN resulted in the nitration of both rings affording 2-(4-hydroxy-3nitrophenyl)-4-nitro-5-pyrimidinol (2u) in 79\% yield. Treatment of $\mathbf{1 x}$ with only one equiv of CAN gave only $\mathbf{2 u}$ in $10 \%$ yield; no mono nitrated derivative was obtained.

We subsequently found that anisole, 2,6-dimethoxy phenol, aniline and $p$-anisidine did not react with $\mathrm{CAN} / \mathrm{NaHCO}_{3}$, indicating that a hydroxyl group and an unsubstituted carbon ortho to the phenol are required for nitration. Several dihydroxyarenes, aromatic amines, and aminophenols were also not nitrated by $\mathrm{CAN} / \mathrm{NaHCO}_{3}$ reagent, whereas the dihydroxyarenes and aminophenols upon addition of CAN were rapidly oxidized giving mixture of products. Furthermore, phenols possessing strongly deactivating groups (in the electrophilic aromatic sense), i.e. 4-cyanophenol and 2-chloro-4-nitrophenol, resisted nitration even when stirred overnight. Interestingly, 3-pyridol (1v) did undergo nitration with stirring for $6 \mathrm{~h}$ in refluxing acetonitrile to give 2-nitro- 3-pyridol (2v) in 76\% yield.

The high ortho regioselectivity observed in the CAN nitration suggest that a Fries type rearrangement may be involved in the nitration. A possible mechanism is shown in Scheme 1. As shown the hydroxy group of the phenol reacts with CAN to give complex $\mathbf{3}$ from which a nitro 
group is transferred from the CAN moiety to the 2-position of the phenol. The resulting intermediate 4 then undergoes aromatization of the ring and the elimination of $\left[\left(\mathrm{ONO}_{2}\right)_{5} \mathrm{CeOH}\right]^{-}$ ${ }^{2}$. In conclusion, a high-yield, regioselective ortho nitration of a wide range of phenols using the inexpensive and easy to use CAN reagent has been demonstrated. It should become an important tool in organic synthesis.

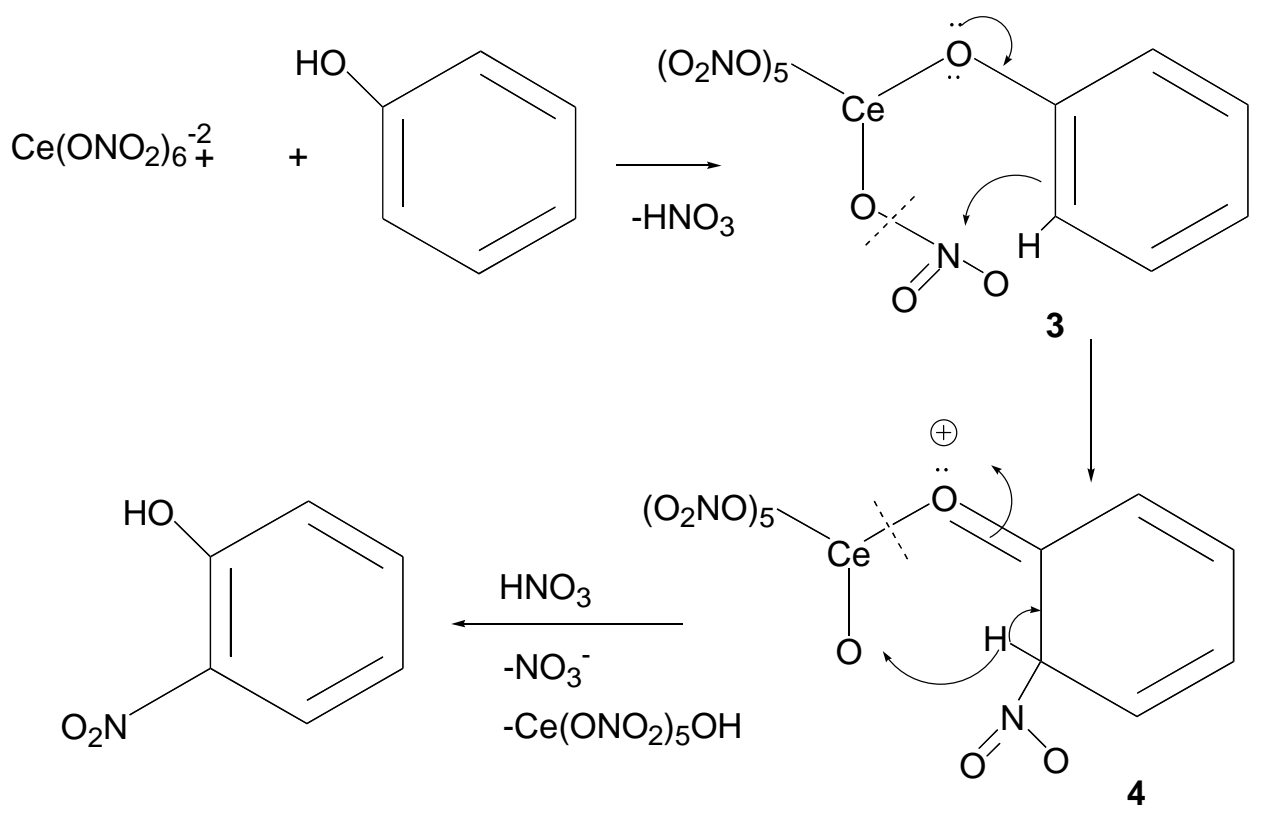

Scheme 1. Possible mechanism for the ortho nitro of phenol.

\section{Experimental Section}

General Procedures. Melting points were in open capillaries and are uncorrected. All reactions were carried out under an atmosphere of dry nitrogen. Phenols (1a-v), CAN, and acetonitrile were purchased from commercial sources. Reactions were monitored by GC/MS. Concentrations were performed by rotary evaporator using water aspirator system. Low-pressure chromatography was carried out by applying air pressure to Pyrex columns packed with silica gel 60 (0.040-0.063 mm particle size, 230-400 mesh). Routine ${ }^{1} \mathrm{H}$ NMR were recorded on a FT NMR at $400 \mathrm{MHz}$ and ${ }^{13} \mathrm{C}$ NMR spectra were recorded at $100 \mathrm{MHz}$.

Experimental procedure. CAN (3.84 g, $7.0 \mathrm{mmol})$ was added to a stirred mixture containing $3.5 \mathrm{mmol}$ of appropriate phenol (1a-x), $\mathrm{NaHCO}_{3}(1.0 \mathrm{~g})$ and $40 \mathrm{~mL}$ of anhydrous $\mathrm{MeCN}$ at $\mathrm{rt}$. The resulting mixture was stirred for 30 min during which time the yellow color of CAN was discharged. The mixture was filtered, washed with water, and extracted with $\mathrm{CHCl}_{3}\left(3 \mathrm{X}_{2} 20\right.$ $\mathrm{mL})$. The combined $\mathrm{CHCl}_{3}$ extracts were dried $\left(\mathrm{Na}_{2} \mathrm{SO}_{4}\right)$, and the solvent evaporated in vacuo to give the corresponding 2-nitrophenol (2). Prior to purification of 2, an aliquote was subjected to 
GC/MS analysis which showed, in all successful reactions, the absence of the 4-nitrophenol positional isomer. The 2-nitrophenol was purified by column chromatography using hexaneethyl acetate $(9: 1)$ as eluent. The physical properties of 1a-w are given below.

2-Nitrophenol (2a). ${ }^{23}$ Yellow solid, mp $44-45{ }^{\circ} \mathrm{C}$ (lit. ${ }^{24} 44.9{ }^{\circ} \mathrm{C} .{ }^{1} \mathrm{H}$ NMR $\left(\mathrm{CDCl}_{3}\right) \delta 6.98(\mathrm{t}, J=$ $1 \mathrm{H}), 7.14(\mathrm{~d}, J=, 1 \mathrm{H}), 7.54(\mathrm{t}, J=, 1 \mathrm{H}), 7.94(2, J=, 1 \mathrm{H}), 10.66$ (brs, $1 \mathrm{H}) .{ }^{13} \mathrm{C} \mathrm{NMR}\left(\mathrm{CDCl}_{3}\right) \delta$ 120.3, 120.6, 125.3, 134.0, 137.9, 155.4.

4-Methyl-2-nitrophenol (2b). ${ }^{25}$ Yellow solid, mp $35{ }^{\circ} \mathrm{C}$ (lit 34-35 $\left.{ }^{\circ} \mathrm{C}\right)^{26} 1 \mathrm{H}$ NMR $\left(\mathrm{CDCl}_{3}\right) \delta$ $2.36(\sigma, 3 \mathrm{H}), 7.06(\delta, J=8.6 \mathrm{~Hz}, 1 \mathrm{H}), 7.35(\mathrm{dd}, J=8.6 \mathrm{~Hz}, 2.1 \mathrm{~Hz}, 1 \mathrm{H}), 7.73(\mathrm{~d}, J=2.1 \mathrm{~Hz}, 1 \mathrm{H})$, $10.45(\mathrm{~s}, 1 \mathrm{H}) .{ }^{13} \mathrm{C} \mathrm{NMR}\left(\mathrm{CDCl}_{3}\right) \delta . .20 .4,119.9,124.5,130.4,139.0,153.4$.

4-Bromo-2-nitrophenol (2c). ${ }^{27}$ Yellow solid, mp 92-93 ${ }^{\circ} \mathrm{C} .{ }^{28}{ }^{1} \mathrm{H}$ NMR $\left(\mathrm{CDCl}_{3}\right) \delta 7.09(\delta, J=$ $8.9 \mathrm{~Hz}, 1 \mathrm{H}), 7.65(\mathrm{dd}, J=8.9 \mathrm{~Hz}, 2.4 \mathrm{~Hz}, 1 \mathrm{H}), 8.24(\mathrm{~d}, J=2.4 \mathrm{~Hz}, 1 \mathrm{H}), 10.51(\mathrm{~s}, 1 \mathrm{H}) .{ }^{13} \mathrm{C}$ NMR $\left(\mathrm{CDCl}_{3}\right) \delta$ 111.1, 121.2, 126.7, 134.0, 139.8, 153.5.

4-Methoxy-2-nitrophenol (2d). Orange solid, mp 79-80 ${ }^{\circ} \mathrm{C}\left(\right.$ lit. $\left.{ }^{29} 83{ }^{\circ} \mathrm{C}\right) .{ }^{1} \mathrm{H}$ NMR $\left(\mathrm{CDCl}_{3}\right) \delta$ 3.85 (s, $3 \mathrm{H}), 7.09(\delta, J=9.2 \mathrm{~Hz}, 1 \mathrm{H}), 7.23(\mathrm{dd}, J=9.2 \mathrm{~Hz}, 3.0 \mathrm{~Hz}, 1 \mathrm{H}), 7.53$ (d, $J=3.0 \mathrm{~Hz}, 1$ $\mathrm{H}), 10.37(\mathrm{~s}, 1 \mathrm{H}) .{ }^{13} \mathrm{C} \mathrm{NMR}\left(\mathrm{CDCl}_{3}\right) \delta .56 .4,106.1,121.3,127.7,134.0,150.5,153.0$.

3-Methoxy-2-nitrophenol (2e). Yellow solid, mp. 52-53 ${ }^{\circ} \mathrm{C}$ (lit. ${ }^{30} 53{ }^{\circ} \mathrm{C}$ ). IR (KBr) 3198, 3020, 1621, 1443, 1321, 834, 757, $669 \mathrm{~cm}^{-1} .{ }^{1} \mathrm{H}$ NMR $\left(\mathrm{CDCl}_{3} \delta 3.90\right.$ (s, $\left.3 \mathrm{H}\right), 6.55$, (dd, $J=7.8 \mathrm{~Hz}, 2.1$ $\mathrm{Hz}, 1 \mathrm{H}), 7.28(\mathrm{dd} J=\mathrm{Hz}, 1 \mathrm{H}), 8.07(\mathrm{~d}, J=7.8 \mathrm{~Hz}, 1 \mathrm{H}), 11.07 .{ }^{13} \mathrm{C} \mathrm{NMR}\left(\mathrm{CDCl}_{3}\right) \delta 56.5$, 101.8, 109.8, 127.4, 128.1, 158.3, 167.5.

5-Bromo-2-nitrophenol (2f). Light yellow solid, mp $44-45{ }^{\circ} \mathrm{C}$ (lit. $\left.{ }^{31} 46-47\right) .{ }^{1} \mathrm{H}$ NMR $\left(\mathrm{CDCl}_{3}\right) \delta$ $7.15(\mathrm{dd}, J=8.8 \mathrm{~Hz}, 2.0 \mathrm{~Hz}, 1 \mathrm{H}), 7.39(\mathrm{~d}, J=2.0 \mathrm{~Hz}, 1 \mathrm{H}), 7.99(\mathrm{dd}, J=8.0 \mathrm{~Hz}, 1 \mathrm{H}) .{ }^{13} \mathrm{C}$ NMR $\left(\mathrm{CDCl}_{3}\right) \delta .123 .4,124.3,126.5,132.8,124.1,155.7$.

5-Methyl-2-nitrophenol (2g): Yellow solid, mp. $54-55$ (lit. ${ }^{32}$ 55-56 ${ }^{\circ} \mathrm{C}$ ). IR (KBr) 3192, 3117, 1927, 1785, 1627, 1479, 1366, 823, 659, $671 \mathrm{~cm}^{-1} .{ }^{1} \mathrm{H} \mathrm{NMR}\left(\mathrm{CDCl}_{3}\right) 2.41(\mathrm{~s}, 3 \mathrm{H}), 6.81(\mathrm{~d}, J=$ $8.7 \mathrm{~Hz}, 1 \mathrm{H}), 6.96$ (brs, $1 \mathrm{H}), 8.00(\mathrm{~d}, J=8.17 \mathrm{~Hz}, 1 \mathrm{H}) .{ }^{13} \mathrm{C} \mathrm{NMR}\left(\mathrm{CDCl}_{3}\right) 22.2,130.0,122.0$, $125.2,132.1,150.2,155.5$.

5-Chloro-2-nitrophenol (2h). Yellow solid mp 39-40 ${ }^{\circ} \mathrm{C}$ (lit. ${ }^{33} 42-43{ }^{\circ} \mathrm{C}$ ). IR (KBr) 3192, 3106, 1912, 1774, 1616, 1460, 1317, 918, 753, $675 \mathrm{~cm}^{-1} .{ }^{1} \mathrm{H}$ NMR $\left(\mathrm{CDCl}_{3}\right) \delta 6.99(\mathrm{dd}, J=9.0 \mathrm{~Hz}, 2.1$ $\mathrm{Hz}, 1 \mathrm{H}), 7.21(\mathrm{~d}, J=2.1 \mathrm{~Hz}, 1 \mathrm{H}), 8.08(\mathrm{~d}, J=9.0 \mathrm{~Hz}, 1 \mathrm{H}), 10.68(\mathrm{~s}, 1 \mathrm{H}) .{ }^{13} \mathrm{C} \mathrm{NMR}\left(\mathrm{CDCl}_{3}\right)$ $120.2,121.4,126.6,132.7,144.1,155.8$.

6-Chloro-4-methoxy-2-nitrophenol (2i). Orange solid, mp 92-93 ${ }^{\circ} \mathrm{C}$. IR (KBr) 3246, 3106, 1753, 1686, 1416, 1248, $761 \mathrm{~cm}^{-1} .{ }^{1} \mathrm{H} \mathrm{NMR}\left(\mathrm{CDCl}_{3}\right) \delta 3.85(\mathrm{~s}, 3 \mathrm{H}), 7.38(\mathrm{~d}, J=3.1 \mathrm{~Hz}, 1 \mathrm{H})$, $7.50(\mathrm{~d}, J=3.1 \mathrm{~Hz}, 1 \mathrm{H}), 10.71(\mathrm{~s}, 1 \mathrm{H}) .{ }^{13} \mathrm{C} \mathrm{NMR}\left(\mathrm{CDCl}_{3}\right) \delta 57.6,105.9,125.5,127.0,134.3$, 146.7, 152.0 .

Anal. Calcd for $\mathrm{C}_{7} \mathrm{H}_{6} \mathrm{ClNO}_{4}$ : C, 41.30; H, 2.97; N, 6.88. Found: C, 41.40; H, 3.01; N, 6.76.

6-Chloro-4-methyl-2-nitrophenol (2j). Yellow solid, mp 65-66 ${ }^{\circ} \mathrm{C}$. IR (KBr) 3190, 3071, 1619, 1464, 1311, 797, 763, 753, $664 \mathrm{~cm}^{-1} .{ }^{1} \mathrm{H}$ NMR $\left(\mathrm{CDCl}_{3}\right) \delta 2.36(\mathrm{~s}, 3 \mathrm{H}), 7.54(\mathrm{~d}, J=1.7 \mathrm{~Hz}, 1 \mathrm{H})$, $7.86(\mathrm{~d}, J=1.8 \mathrm{~Hz}, 1 \mathrm{H}), 10.86(\mathrm{~s}, 1 \mathrm{H}) .{ }^{13} \mathrm{C} \mathrm{NMR}\left(\mathrm{CDCl}_{3}\right) \delta 20.5,123.5,124.3,130.3,134.3$, 138.8, 149.6. Anal. Calcd for $\mathrm{C}_{7} \mathrm{H}_{6} \mathrm{ClNO}_{3}$ : C, 44.82; H, 3.22; N, 7.47. Found: $\mathrm{C}, 44.89 ; \mathrm{H}, 3.18$; $\mathrm{N}, 7.53$. 
4,5-Dimethyl-2-nitrophenol (2k). Yellow solid, mp $82-84{ }^{\circ} \mathrm{C}\left(\right.$ lit. $\left.^{34} 82-83{ }^{\circ} \mathrm{C}\right) .{ }^{1} \mathrm{H}$ NMR $\left(\mathrm{CDCl}_{3}\right)$ $\delta 2.26(\mathrm{~s}, 3 \mathrm{H}), 2.32(\mathrm{~s}, 3 \mathrm{H}), 6.95(\mathrm{~s}, 1 \mathrm{H}), 7.86(\mathrm{~s}, 1 \mathrm{H}), 10.49(\mathrm{~s}, 1 \mathrm{H}) .{ }^{13} \mathrm{C} \mathrm{NMR}\left(\mathrm{CDCl}_{3}\right) \delta 19.2$, 20.8, 120.4, 125.0, 130.1, 134.1, 149.9, 154.8 .

3,6-Dimethyl-2-nitrophenol (2l). Yellow solid, mp 33-34 ${ }^{\mathrm{O}} \mathrm{C}\left(\right.$ lit. $\left.^{35} 34-35{ }^{\circ} \mathrm{C}\right) .{ }^{1} \mathrm{H}$ NMR $\left(\mathrm{CDCl}_{3}\right)$ $\delta 2.26(\mathrm{~s}, 3 \mathrm{H}), 2.32{ }^{13} \mathrm{C} \mathrm{NMR}\left(\mathrm{CDCl}_{3}\right) \delta 16.2,22.7,123.2,127.0,134.2,135.3,136.4,154.1$.

Methyl 4-hydroxy-3-nitrobenzoate (2m). Yellow solid, mp $76-77{ }^{\circ} \mathrm{C}$ (lit. $\left.{ }^{36} 77-78{ }^{\circ} \mathrm{C}\right) .{ }^{1} \mathrm{H} \mathrm{NMR}$ $\left(\mathrm{DMSO}_{6}\right) \delta 3.85(\mathrm{~s}, 3 \mathrm{H}), 7.23(\mathrm{dd}, J=8.7 \mathrm{~Hz}, 1.7 \mathrm{~Hz}, 1 \mathrm{H}), 8.06(\mathrm{dd}, J=8.7 \mathrm{~Hz}, 1.7 \mathrm{~Hz}, 1 \mathrm{H})$, 8.39 (d, $J=1.7 \mathrm{~Hz}, 1 \mathrm{H}) .{ }^{13} \mathrm{C}$ NMR 53, 120.2, 121.2, 127.6, 136.0, 137.5, 156.7, 165.4.

Methyl 3-hydroxy-4-nitrobenzoate: (2n). Yellow solid, mp. 91-92 ${ }^{\mathrm{O}} \mathrm{C}$. IR (KBr) 3317, 3123, 2961, 1724, 1624, 1478, 1326, 844, 747, $688 \mathrm{~cm}^{-1}{ }^{1} \mathrm{H}$ NMR $\left(\mathrm{CDCl}_{3}\right) \delta 3.98$ (s, $\left.3 \mathrm{H}\right), 7.64$ (dd, $J$ $=8.8 \mathrm{~Hz}, 1.76 \mathrm{~Hz}, 1 \mathrm{H}), 7.86(\mathrm{~m}, 1 \mathrm{H}), 8.20(\mathrm{dd}, J=8.8 \mathrm{~Hz}, 2.0 \mathrm{~Hz}, 1 \mathrm{H}){ }^{13} \mathrm{C} \mathrm{NMR}\left(\mathrm{CDCl}_{3}\right) \delta$ $53.3,121.0,122.0,125.6,136.2,138.2,155.0,165.2$ :

3-Hydroxy-4-nitrobenzaldehyde (2o). Yellow solid, mp. $127-128{ }^{\mathrm{O}} \mathrm{C}$ (lit. $\left.{ }^{33} 128{ }^{\circ} \mathrm{C}\right) . \mathrm{IR}(\mathrm{KBr})$ 3260, 3087, 2877, 1700, 1621, 1478, 1315, 759, 704. ${ }^{1} \mathrm{H} \mathrm{NMR}\left(\mathrm{CDCl}_{3}\right) \delta .7 .52$ (dd, $J=8.6 \mathrm{~Hz}$, 2.1Hz, $1 \mathrm{H}), 7.67(\mathrm{~d}, J=2.1 \mathrm{~Hz}, 1 \mathrm{H}), 8.30(\mathrm{dd}, J=8.6 \mathrm{~Hz}, 2.1 \mathrm{~Hz}, 1 \mathrm{H}), 10.0(\mathrm{~s}, 1 \mathrm{H}), 10.6$ (s, $1 \mathrm{H}) .{ }^{13} \mathrm{C} \mathrm{NMR}\left(\mathrm{CDCl}_{3}\right) \delta 119.7,122.4,125.6,136.8,142.6,155.8,190.5$.

2-Nitro-1-naphthol (2p). ${ }^{37}$ Yellow solid, mp 126-127 ${ }^{\circ} \mathrm{C}$ (lit. $\left.{ }^{38} 125-127{ }^{\circ} \mathrm{C}\right) .{ }^{1} \mathrm{H}$ NMR $\left(\mathrm{CDCl}_{3}\right) \delta$ $7.27(\mathrm{~d}, J=9.2 \mathrm{~Hz}, 1 \mathrm{H}), 7.61(\mathrm{tt}, J=7.5 \mathrm{~Hz}, 1.5 \mathrm{~Hz}, 1 \mathrm{H}), 7.70(\mathrm{tt}, J=7.5 \mathrm{~Hz}, 1.5 \mathrm{~Hz}, 1 \mathrm{H}), 7.80$ (d, $9.2 \mathrm{~Hz}, 1 \mathrm{H}), 7.99$ (d, $J=9.2 \mathrm{~Hz}, 1 \mathrm{H}), 8.53$ (d, $J=9.2 \mathrm{~Hz}, 1 \mathrm{H}), 12.26(\mathrm{~s}, 1 \mathrm{H}) .{ }^{13} \mathrm{C}$ NMR $\left(\mathrm{CDCl}_{3}\right) \delta 119.8,120.6,125.5,127.2,127.5,128.7,131.8,134.1,137.8,156.1$.

1-Nitro-2-naphthol (2q). Yellow solid, mp, 95-96 ${ }^{\circ} \mathrm{C}\left(\right.$ lit. $\left.^{33} 96{ }^{\mathrm{O}} \mathrm{C}\right) .{ }^{1} \mathrm{H}$ NMR $\left(\mathrm{CDCl}_{3}\right) \delta 7.28$ (d, $J=9.1 \mathrm{~Hz}, 1 \mathrm{H}), 7.53(\mathrm{tt}, 7.6 \mathrm{~Hz}, 1.8 \mathrm{~Hz}, 1 \mathrm{H}), 7.76(\mathrm{tt}, 7.6 \mathrm{~Hz}, 1.8 \mathrm{~Hz}, 1 \mathrm{H}), 7.85(\mathrm{~d}, J=9.1 \mathrm{~Hz}$, ), $8.01(\mathrm{~d}, J=9.1 \mathrm{~Hz}, 1 \mathrm{H}), 8.94(\mathrm{~d}, J=9.1 \mathrm{~Hz}, 1 \mathrm{H}), 12.21(\mathrm{~s}, 1 \mathrm{H}) .{ }^{13} \mathrm{C} \mathrm{NMR}\left(\mathrm{CDCl}_{3}\right) \delta 119.7$, $123.4,126.0,127.1,128.3,128.9,129.6,131.3,139.5,159.2$.

7-Hydroxy-3,4,8-trimethyl-6-nitrocoumarin (2r). ${ }^{1} \mathrm{H}$ NMR $\left(\mathrm{CDCl}_{3}\right) 2.24(\mathrm{~s}, 3 \mathrm{H}), 2.42(\mathrm{~s}, 1$ $\mathrm{H}), 2.44(\mathrm{~s}, 1 \mathrm{H}), 8.31(\mathrm{~s}, 1 \mathrm{H}), 11.15(\mathrm{~s}, 1 \mathrm{H}) .{ }^{13} \mathrm{C} \mathrm{NMR}\left(\mathrm{CDCl}_{3}\right) \delta$ 8.8, 13.8, 15.6, 114.6, 116.2, 119.5, 122.1, 130.6. 145.4, 155.1, 156.0, 161.0. MS m/z $278\left(\mathrm{M}^{+}\right)$. Anal. Calcd for $\mathrm{C}_{12} \mathrm{H}_{11} \mathrm{NO}_{5}$ : C, 57.83; H, 4.45; N, 5.62. Found: C, 57.90; H, 4.50; N, 5.66.

6-Hydroxy-5-nitro-1,3-benzoxanthio-2-one (2s). Yellow solid, mp 178-180 ${ }^{\mathrm{O}} \mathrm{C}$. IR ( $\left.\mathrm{KBr}\right)$ 3282, 3010, 1758, 1624, 1447, 1321, 891, 811, $742 \mathrm{~cm}^{-1} .{ }^{1} \mathrm{H}$ NMR (DMSO-d $)_{6} \delta 7.18$ (s, $1 \mathrm{H}$ ), 8.45 (s, $1 \mathrm{H}), 11.54(\mathrm{~s}, 1 \mathrm{H})$. MS m/z $213 \mathrm{M}^{+} .{ }^{13} \mathrm{C}$ NMR DMSO-d 6 $\delta 100.6,111.7,119.4,132.7$, 150.4, 151.7, 167.7. MS m/z Anal. Calcd for $\mathrm{C}_{7} \mathrm{H}_{3} \mathrm{NO}_{5} \mathrm{~S}: \mathrm{C}, 39.44 ; \mathrm{H}, 1.42 ; \mathrm{N}, 6.57$. Found: $\mathrm{C}$, $39.37 ; \mathrm{H}, 1.46 ; \mathrm{N}, 6.55$.

2-(2-Hydroxy-3-nitrophenyl)benzoxazole (2t). Colorless solid, mp 195-197 ${ }^{\circ} \mathrm{C}$. IR (KBr) ${ }^{1} \mathrm{H}$ NMR (DMSO-d $\left.)_{6}\right) \delta .33(\mathrm{~m}, 1 \mathrm{H}), 7.49(\mu, 2 \mathrm{H}), 7.91(\mu, 2 \mathrm{H}), 8.33(\mathrm{~m}, 1 \mathrm{H}), 8.38(\mathrm{t}, \mathrm{J}=2.8 \mathrm{~Hz}$, $1 \mathrm{H}) .{ }^{13} \mathrm{C} \mathrm{NMR}\left(\mathrm{CDCl}_{3}\right) \delta 110.9,111.5,118.6,120.0,124.0,126.1,126.9,128.9,139.7,140.9$, 149.6, 161.3, 163.7. MS m/z $256\left(\mathrm{M}^{+}\right)$: Anal. Calcd for $\mathrm{C}_{13} \mathrm{H}_{8} \mathrm{~N}_{2} \mathrm{O}_{4}: \mathrm{C}, 60.94 ; \mathrm{H}, 3.15 ; \mathrm{N}, 10.93$. Found: C, 61.91; H, 3.15; N, 10.85 .

2-(4-Hydroxy-3-nitrophenyl)-4-nitro-5-pyrimidinol (2u). Yellow solid, mp. 220-223 ${ }^{\circ} \mathrm{C}$. IR (KBr) 3322, 2201, 1630, 1440, $1321 \mathrm{~cm}^{-1} .{ }^{1} \mathrm{H}$ NMR (DMSO-d $\left.)\right) \delta .27$ (d, J = 7.6 Hz, $\left.1 \mathrm{H}\right), 8.35$ 
(dd, 7.6, $1.7 \mathrm{~Hz}, 1 \mathrm{H}), 8.67(\mathrm{~d}, J=1.7 \mathrm{~Hz}, 1 \mathrm{H}), 8.93(\mathrm{~s}, 1 \mathrm{H}) .{ }^{13} \mathrm{C}$ NMR $\left(\right.$ DMSO- $\left.d_{6}\right) \delta 120.7$, 124.9, 127.4, 134.2, 137.8, 142.8, 151.3, 152.5, 153.9, 154.8. MS m/z $278\left(\mathrm{M}^{+}\right)$. Anal. Calcd for $\mathrm{C}_{10} \mathrm{H}_{7} \mathrm{~N}_{3} \mathrm{O}_{4}: \mathrm{C}, 51.51 ; \mathrm{H}, 3.03 ; \mathrm{N}, 18.02$. Found: $\mathrm{C}, 51.66 ; \mathrm{H}, 3.09 ; \mathrm{N}, 18.12$.

3-Hydroxy-2-nitropyridine (2v). Yellow solid, mp $66-68{ }^{\circ} \mathrm{C}$, (lit.67-69 $\left.{ }^{\circ} \mathrm{C}\right) .{ }^{39}$

\section{Acknowledgements}

We thank the Welch Foundation, Houston, TX for financial support.

\section{References and Notes}

1. Zeegers, P. J. J. Chem. Edu. 1993 70, 1036.

2. Thompson, M. J.; Zeegers. P. J. Tetrahedron 1991, 47, 8787 and references therein.

3. Iranpoor, N.; Firouzabadi, H.; Heydari; R. Synth. Commun. 1999, 29, 3295.

4. Zolfigol, M. A.; Madrakian, E.; Ezat, G. Indian. J. Chem. 2001, 40B, 1191.

5. Roussel, J.; Lemaire, M.; Guy, M.; Guette, J. P. Tetrahedron Lett. 1986, $27,27$.

6. Frechette, R. F.; Beach, M. J. Synth. Commun. 1998, 28, 3471.

7. Sato, Y.; Yamada, M.; Yoshida, S.; Soneda, T.; Ishikawa, M.; Nizato, T.; Suzuki, K.; Konno, F. J. Med. Chem. 1998, 41, 3015.

8. Humayun, P.; Samuel, O. O.; Lilias, R.; James, R. R.; Colin, J. S. Tetrahedron 1988, 44, 4555.

9. Gigante, B.; Prazeres, A. O.; Marcelo-Curto, M. J.; Lazlo, P. J. Org. Chem. 1995, 60, 3445.

10. Ganguly, N.; Sukai, A. K.; De, S. Synth. Commun. 2001, 31, 301.

11. Chawla, H. M.; Sharma, S. K.; Chakrabarty, K.; Bhanumati, S. J. Chem. Soc. Chem, Commun. 1988, 2, 128.

12. Chaudhuri, K. J; Chawla, H. M. Indian. J. Chem. 1985, 24B, 1184.

13. Strauss, C. R.; Trianor, R. W. Aust. J. Chem. 1995, 48, 1665.

14. Majetich, G.; Hicks, R. Radiat. Phys. Chem. 1995, 45, 567.

15. Grenier, J.-L.; Catteau, J. P.; Cotelle, P. Synthetic Commun. 1999, 29, 1201.

16. Tale, R. H. Org. Lett. 2002, 4, 1641.

17. Chaudhuri, K.; Chawla, H. M. Indian. J. Chem. 1985, 24B, 1277.

18. Chakrabarty, K.; Chawla, H. M.; Suresh, V. V. Indian. J. Chem. 1992, 31B, 464.

19. Maleski, R. J.; Kluge, M.; Sicker, D. Synthetic Commun. 1995, 25, 2327.

20. Fischer, A.; Mathivanan, N. Tetrahedron Lett. 1988, 29, 1869.

21. Lemaire, M.; Guy, A, Roussel, J.; Guette, J.-P. Tetrahedron 1987, 43, 835.

22. Chawla, H. M; Mittal, R. S. Synthetic Commun. 1985, 70.

23. The Aldrich Library of Spectra: ${ }^{1} \mathrm{H}$ and ${ }^{13} \mathrm{C}$ NMR spectra 3, 682C.

24. Hart, R. S. J. Am. Chem. Soc. 1910, 32, 1105. 
25. The Aldrich Library of Spectra: ${ }^{1} \mathrm{H}$ and ${ }^{13} \mathrm{C}$ NMR spectra 2, 731C.

26. Barrett, J. N.; Moodie, R. B.; Scholefield, K.; Weston, J. B. J. Chem. Soc. Perk. Trans. II 1977, 248.

27. The Aldrich Library of Spectra : ${ }^{1} \mathrm{H}$ and ${ }^{13} \mathrm{C}$ NMR spectra 2, 735B. Erp, M. H. Recl. Trav. Chim. Pays-Bas 1920, 29, 187.

28. Jenes, S.; Messeguer, A. Tetrahedron 1999, 55, 14111.

29. Carpenter, M. S.; Easter, W. M.; Wood, T. F. J. Org. Chem. 1951, 16, 586.

30. Mangini, A. Gazz. Chim. Ital. 1936, 66, 675.

31. Gibson, G. P. J. Chem. Soc. 1923, 123, 1269.

32. Hodgson, H. H.; Moore, F. H. J. Chem. Soc. 1925, 127, 875.

33. Linderberg, M.; Hellberg, S.; Bjork, S.; Gotthammar, B.; Hogberg, T.; Persson, K.; Schwarcz, R.; Luthman, J.; Johansson, R. Eur. J. Med. Chem. 1999, 34, 729.

34. Holler, A. C.; Huggett, C.; Rathmann, F. R. J. Am. Chem. Soc. 1950, 72, 2034.

35. Einhorn, A. Liebigs Ann. Chem. 1900, 311, 26.

36. The Aldrich Library of Spectra : ${ }^{1} \mathrm{H}$ and ${ }^{13} \mathrm{C}$ NMR spectra $2,783 \mathrm{C}$.

37. Edwards, Jr., W. R.; Tate, C. W. Anal. Chem. 1951, 23, 826.

38. De Selms, R. C. J. Org. Chem. 1968, 33. 478. 